\title{
Interferone schützen bei Multipler Sklerose vor schwerem COVID-19-Verlauf
}

Eine MS-Therapie mit Interferon senkt das Risiko für einen schweren COVID-19-Verlauf um zwei Drittel, CD-20-Antikörper verdoppeln es hingegen. Insgesamt erhöht eine Multiple Sklerose das Risiko für schwere Verläufe deutlich.

\begin{abstract}
Zu Beginn der COVID-19-Pandemie lieferten MS-Register rasch ein Bild über die Gefährdung von MS-Patienten: Zum einen gelten hier auch die in der Allgemeinbevölkerung üblichen Risikofaktoren für einen schweren Verlauf wie höheres Alter, Übergewicht oder kardiometabolische Erkrankungen, zum anderen können immunmodulierende MS-Therapien den Verlauf beeinflussen. Hier deutete sich - wenig überraschend - ein erhöhtes Risiko für COVID-19-Komplikationen bei B-Zell-depletierender MS-Therapie an, gleichzeitig ergaben sich in einigen Untersuchungen protektive Effekte für MS-Patienten mit Interferonen und anderen Basistherapeutika. So hatten Ärzte auf dem Kongress der europäischen MS-Gesellschaft ECTRIMS vor einem Jahr globale Registerdaten vor-
\end{abstract}

gestellt, nach denen sich das Risiko für einen schweren COVID-19-Verlauf unter CD-20-Antikörpern um 50-100\% erhöht, die Resultate waren aber nicht in allen Registern konsistent. Für einige Basistherapeutika deutet sich wiederum ein reduziertes Risiko für schwere Verläufe im Vergleich zu MS-Kranken ohne krankheitsmodifizierende Arzneien (DMD) an. Allerdings sei die Datenerfassung von COVID-19-Kranken in den MSRegistern in den ersten Pandemiemonaten recht chaotisch gewesen, so die MS-Spezialistin Prof. Dr. Maria Pia Sormani von der Universität Genua auf der aktuellen ECTRIMS-Tagung. Oft sei COVID-19 nur bei schwer kranken MS-Patienten erfasst worden, was die Resultate verzerrt habe. Seit dem Sommer 2020 würden in vielen
Ländern MS-Kranke regelmäßig auf SARS-CoV-2 getestet, sodass man nun einen wesentlich besseren Überblick habe, wie häufig schwere Verläufe bei MS-Kranken auftreten.

Sormani verwies auf aktuelle Resultate des italienischen MS-Registers, das einen großen Teil der MS-Kranken des Landes erfasst. Eine Auswertung von knapp 1600 MS-Patienten mit nachgewiesener SARS-CoV-2-Infektion bestätigt ein um $90 \%$ erhöhtes Risiko für einem schweren COVID-19-Verlauf mit Pneumonie und Hospitalisierung unter $C D$-20-Antikörpern wie Ocrelizumab und Rituximab - verglichen mit MS-Patienten ohne DMD, aber ähnlichem Alter und Begleiterkrankungen. Das Risiko scheint unter Rituximab stärker ausgeprägt zu sein, allerdings sind diese Zahlen nicht sehr

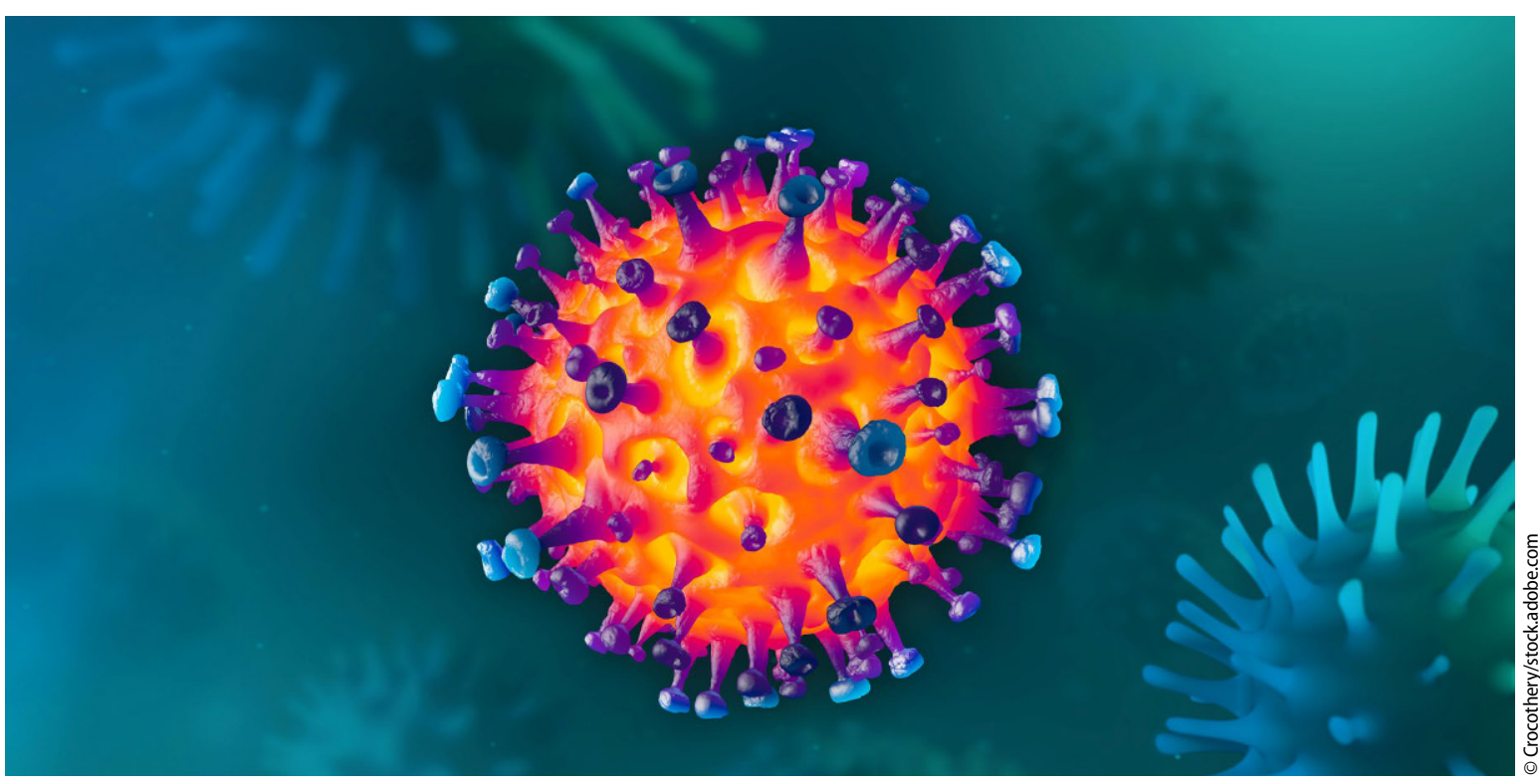

A Multiple Sklerose erhöht das Risiko für schwere COVID-19-Verläufe deutlich 
belastbar, da nur wenige MS-Patienten damit behandelt worden sind. Ein klarer Zusammenhang ergibt sich jedoch mit der Dauer der Therapie: Je länger die Patienten mit CD-20-Antikörpern behandelt worden sind, umso höher ist das Risiko für einen schweren COVID-19-Verlauf.

\section{Hohes Risiko unter Hochdosis- \\ Kortikoidtherapie}

Ungünstig scheint auch eine Hochdosis-Kortikoidtherapie vor der Infektion zu sein: Für MS-Patienten mit einer solchen Behandlung, etwa aufgrund eines MS-Schubs, ergibt sich ein 2,3fach erhöhtes Risiko für einen schweren COVID-19-Verlauf.

Zugleich bestätigt die Analyse die vor einem Jahr in einer französischen Untersuchung bemerkte Risikoreduktion unter Interferonen: Die Gefahr für einen schweren Verlauf ist nach diversen Adjustierungen nur etwa ein Drittel so hoch wie bei MS-Kranken ohne DMD - ein statistisch signifikanter
Unterschied. „Tatsächlich haben wir in Italien in der Pandemie praktisch keine MS-Patienten mit Interferonen auf einer Intensivstation gesehen, und das liegt nicht an der geringen Zahl der Patienten, solche Medikamente erhalten hier noch etwa $20 \%$ ", erläuterte Sormani. Eine Erklärung könnten die antiviralen Effekte von Interferonen liefern.

Für Teriflunomid ergibt sich eine Risikoreduktion um etwa $50 \%$, allerdings wurde in der Analyse das Niveau für eine statistische Signifikanz knapp verfehlt.

Eine gepoolte Analyse italienischer und französischer Registerdaten bestätigt die Ergebnisse: Das Risiko für einen schweren Verlauf ist danach unter CD-20-Antikörpern verdoppelt, unter Hochdosis-Methylprednisolon 2,7fach höher und unter Interferonen um $58 \%$ geringer als bei einer Therapie mit anderen DMD.

Unbereinigt liegt die Rate schwerer COVID-19-Verläufe bei MS-Patienten auf dem Niveau der Allgemeinbevöl- kerung: Die Sterberate unter bestätigten Fällen erreicht in den italienischen Registerdaten 1,6\%. Da unter den MSPatienten aber der Anteil recht junger Personen und Frauen überwiegt, ergibt sich alters- und geschlechtsadjustiert ein etwa verdoppeltes Risiko für eine Hospitalisierung, ein knapp dreifach erhöhtes Risiko für eine Intensivbehandlung sowie eine zweieinhalbfach erhöhte Mortalität.

www.springermedizin.de/ Thomas Müller

Hinweis des Verlags. Der Verlag bleibt in Hinblick auf geografische Zuordnungen und Gebietsbezeichnungen in veröffentlichten Karten und Institutsadressen neutral.

psychopraxis. neuropraxis $2022 \cdot 25: 10-11$ https://doi.org/10.1007/s00739-02200784-x

(C) The Author(s), under exclusive licence to Springer-Verlag GmbH Austria, ein Teil von Springer Nature 2022
Quelle: 37th Congress of the European Committee for Treatment and Research in Multiple Sclerosis (ECTRIMS); 13.-15. October 2021. Session: Hot Topic 2: COVID-19 and MS. Maria Pia Sormani: Lessons learned from COVID-19 \& MS registries and data gaps.

Hier steht eine Anzeige. 